\title{
Modeling of levothyroxine in newborns and infants with congenital hypothyroidism: challenges and opportunities of a rare disease multi- center study
}

\author{
Gilbert Koch $^{1}$ (1) Britta Steffens ${ }^{1} \cdot$ Stephanie Leroux $^{1} \cdot$ Verena Gotta $^{1} \cdot$ Johannes Schropp $^{2}$. \\ Pascal Gächter ${ }^{1,6} \cdot$ Freya Bachmann $^{2} \cdot$ Tatjana Welzel $^{1} \cdot$ Marco Janner $^{3} \cdot$ Dagmar L'Allemand $^{4}$. \\ Daniel Konrad $^{5}$ (D) $\cdot$ Gabor Szinnai ${ }^{6,7}$ (D) $\cdot$ Marc Pfister $^{1,7}$
}

Received: 16 December 2020/Accepted: 12 May 2021 / Published online: 11 June 2021

(C) The Author(s) 2021

\begin{abstract}
Modeling of retrospectively collected multi-center data of a rare disease in pediatrics is challenging because laboratory data can stem from several decades measured with different assays. Here we present a retrospective pharmacometrics (PMX) based data analysis of the rare disease congenital hypothyroidism $(\mathrm{CH})$ in newborns and infants. Our overall aim is to develop a model that can be applied to optimize dosing in this pediatric patient population since suboptimal treatment of $\mathrm{CH}$ during the first 2 years of life is associated with a reduced intelligence quotient between 10 and 14 years. The first goal is to describe a retrospectively collected dataset consisting of 61 newborns and infants with $\mathrm{CH}$ up to 2 years of age. Overall, 505 measurements of free thyroxine (FT4) and 510 measurements of thyrotropin or thyroid-stimulating hormone were available from patients receiving substitution treatment with levothyroxine (LT4). The second goal is to introduce a scale/location-scale normalization method to merge available FT4 measurements since 34 different postnatal age- and assay-specific laboratory reference ranges were applied. This method takes into account the change of the distribution of FT4 values over time, i.e. a transformation from right-skewed towards normality during LT4 treatment. The third goal is to develop a practical and useful PMX model for LT4 treatment to characterize FT4 measurements, which is applicable within a clinical setting. In summary, a time-dependent normalization method and a practical PMX model are presented. Since there is no on-going or planned development of new pharmacological approaches for CH, PMX based modeling and simulation can be leveraged to personalize dosing with the goal to enhance longer-term neurological outcome in children with the rare disease $\mathrm{CH}$.
\end{abstract}

Keywords Normalization - Reference range - Scale/location-scale P Pediatrics · Pharmacokinetics - Levothyroxine · Congenital hypothyroidism $\cdot$ Rare disease $\cdot$ Thyroid

\section{Introduction}

Analysis and modeling of retrospective clinical data are important to characterize a patient cohort under treatment and to model general and individual disease progression to quantitatively describe unsolved clinical problems, e.g. in a dose- or disease severity-dependent way. Especially for rare diseases, defined as condition that affects less than 200 000 people in the US [1] or less than 1 out of 2000 people

Gilbert Koch and Britta Steffens are shared first authors; Gabor Szinnai and Marc Pfister shared last authors.

Extended author information available on the last page of the article in the European Union [2], retrospective data analysis of patients diagnosed during a time window of 10 to 20 years is crucial for overcoming the limitation of the low number of patients in the general population. Rigorous retrospective analyses pave the way to successful planning of prospective studies. For rare diseases, there is often no ongoing or planned development of new pharmacological approaches. Hence, personalized dosing based on pharmacometric (PMX) modeling and simulation $[3,4]$ is the next logical step to enhance medical treatment in patients with a rare disease. However, before general application of such PMX models, their accuracy needs to be validated in prospective controlled studies. 
PMX modeling of retrospectively collected data of a rare disease originating from several decades and different centers is challenging. First, measurements of one specific laboratory parameter were performed with different commercially available laboratory assays at different centers. Second, different generations of the same laboratory assay over time in the same center are associated with varying reference ranges. Third, a majority of guidelines for standardized clinical and laboratory follow-up for a specific rare disease were developed during the last 5 to 10 years, which may result in specific clinical and laboratory investigations varying from center to center especially in older datasets.

Here we present retrospective data analysis and modeling of congenital hypothyroidism $(\mathrm{CH})$ which is a rare disease (ORPHA:442) that affects 1 out of approximately 1500 to 4000 newborns $[5,6]$. In patients with primary or thyroidal $\mathrm{CH}$, the thyroid gland does not produce sufficient thyroid hormones leading untreated to growth failure and strongly reduced neurological outcome. Therefore, the manufactured form levothyroxine (LT4) of the thyroid hormone thyroxine (T4) is applied to treat thyroid hormone deficiency. $\mathrm{CH}$ is the most frequent preventable cause of mental retardation worldwide. Neurological outcome of $\mathrm{CH}$ patients has been strongly improved in the last 40 years by introducing systematic neonatal screening for preclinical diagnosis in the 1970s [7, 8], and by increasing the starting dose of LT4 at diagnosis from 5-10 to $10-15 \mathrm{mcg} /$ $\mathrm{kg}$ body weight in the 1990s [9, 10]. The main aim was to aggressively correct laboratory hypothyroidism as rapidly as possible to protect thyroid-hormone dependent neurodevelopment in the newborn affected by $\mathrm{CH}$, as during pregnancy, hypothyroidism of fetuses affected by $\mathrm{CH}$ is only partially compensated by transplacental passage of maternal thyroid hormones [11]. Recent data however revealed frequent long-lasting overdosing of patients under the recommended initial dose of $10-15 \mathrm{mcg} / \mathrm{kg}$ body weight $[12,13]$. Finally, different studies showed a negative effect of long-term elevated plasma thyroxine levels during the first years of life on the intelligence quotient between 10 and 14 years [14-16]. Therefore, it is essential to develop a mathematical PMX model to characterize individual dynamics of substituted thyroxine and to further optimize and personalize LT4 treatment in the context of rapid weight gain during infancy and three disease severity levels (mild, moderate and severe $\mathrm{CH}$ disease according to current guidelines) [5].

This article has three goals. First, a retrospectively collected dataset consisting of $n=61$ newborns and infants with $\mathrm{CH}$ up to 2 years of age is described. Second, due to the multi-center/-assay nature of the data, a scale/locationscale normalization method is developed to make the measured free T4 (FT4) concentrations comparable by normalization to a target reference range. For normalization, it is essential that underlying statistical assumptions of the methods are fulfilled in the dataset. Since FT4 measurements change their distribution during treatment, namely from a right-skewed distribution (scale formula) towards normality (location-scale formula), this time-dependent transition of the distribution is taken into account in the developed normalization method. Third, a mathematical model to characterize FT4 measurements and LT4 treatment has to be in a fair balance between the physiological mechanism and the capability to characterize available data. The hypothalamic-pituitary-thyroid (HPT) axis is a complex multi-loop feedback mechanism where, among many others, T4 and thyroid-stimulating hormone (TSH) control themselves to hold all thyroid hormones in a healthy equilibrium. Interestingly, mathematical modeling of the HPT axis has a long history ranging back to the 1950s [17, 18]. However, most mathematical models developed in the last decades are pretty detailed [19-21], based on animal data [22], or focus on a very specific question such as the relationship between TSH and FT4 [23, 24]. Application of such models to data collected in daily clinical routine from $\mathrm{CH}$ patients is usually impossible because many model parameters are not identifiable due to lack of large quantitative data. Therefore, we developed a practical PMX model describing FT4 concentration and LT4 treatment.

In summary, a PMX model for FT4 concentration and LT4 treatment based on normalized FT4 measurements is presented. The normalization method takes the transition from a right-skewed distribution towards normality during LT4 treatment into account. Such a PMX model can be applied to characterize FT4 under LT4 substitution therapy with the goal to further personalize and enhance LT4 treatment in pediatric patients with a rare disease.

\section{Methods}

The Method section consists of six paragraphs. First, study design and retrospective data collection procedure to form the pediatric $\mathrm{CH}$ study population based on data from four different hospitals over the last 25 years are reported. Second, a descriptive analysis of the collected data is presented. Third, normalization procedures for laboratory reference ranges stemming from different assays are introduced. Fourth, a PMX model to characterize FT4 concentration based on remaining endogenous $\mathrm{T} 4$ production and exogenous LT4 administration is presented. Fifth, available covariates are discussed and finally, some remarks about applied statistics and software are presented. 


\section{Study design}

A retrospective multi-center longitudinal cohort study of consecutive pediatric patients diagnosed with primary or thyroidal $\mathrm{CH}$ based on elevated TSH values in neonatal screening and confirmatory laboratory testing was performed between 01/1990 and 08/2018 in Switzerland. Data from neonates and infants up to approximately 2 years of age were included if they (i) had a confirmed diagnosis of primary or thyroidal $\mathrm{CH}$, (ii) were treated at the participating study centers (a) between 01/1990 to 08/2018 (University Children's Hospital Basel, University Hospital Bern, University Children's Hospital Zurich) or (b) between 01/1990 to 12/2013 (Children's Hospital Eastern Switzerland, St. Gallen), (iii) had a complete dataset, including (a) clinical baseline characteristics, (b) laboratory parameters at diagnosis and/or LT4 treatment start, and (c) LT4 dose history during the complete follow-up, (iv) had $\geq 2$ follow-up visits after LT4 treatment start. Patients were excluded, (i) if LT4 start dose and LT4 doses during follow-up visits were missing, (ii) in case of treatment noncompliance, or (iii) in all cases of central hypothyroidism. Data were captured standardized in the designated electronic database secuTrial ${ }^{\circledR}$ for each study visit. An ethical approval for this study (2018-01770) was obtained by the lead local Ethics Committee (Ethikkommission Nordwest- und Zentralschweiz EKNZ) and all local responsible Ethics Committees (Kantonale Ethikkommission Bern, Ethikkommission Zürich, Ethikkommission Ostschweiz EKOS). Data from patients were used pseudonymized. The study was performed in compliance with the Helsinki Declaration and Good Clinical Practice.

\section{Descriptive analysis of the retrospective data}

In total, $\mathrm{n}=71$ pediatric patients with the rare disease $\mathrm{CH}$ fulfilled all inclusion criteria. Clinical records are often stored for approximately 20 years as the patients were monitored from birth until transition to an adult endocrinologist and the retention requirement is 10 years in Switzerland. In addition to the inclusion and exclusion criteria formulated above, only patients with more than 2 months of treatment were included in this analysis. Therefore, the final number of patients involved in the analysis was $\mathrm{n}=61$ (female $=70 \%)$.

Laboratory data from start of LT4 treatment $\left(t_{0}=0\right.$ day $)$ were included in the analysis. Measurements of non-normalized FT4 $(\mathrm{n}=505)$ and TSH $(\mathrm{n}=510)$ are shown in Fig. 1. On average, 8 FT4 measurements were available per patient with minimally 4 and maximally 14 measurements. Disease severity was defined based on the first FT4 measurement at time of diagnosis, i.e. 18 patients were categorized as severe (FT4 $<5 \mathrm{pmol} / \mathrm{l}), 17$ as moderate (FT4 $\geq 5$ and $<10 \mathrm{pmol} / \mathrm{l}$ ) and 21 as mild (FT4 $\geq 10 \mathrm{pmol} / \mathrm{l}$ ) according to current guidelines [5]. Five patients had no initial FT4 measurement. Patient characteristics such as gestational age (GA) as well as postnatal age (PNA), weight, non-normalized FT4 and TSH concentrations, and in addition, total daily LT4 dose and LT4 dose per $\mathrm{kg}$ body weight all at start of treatment and last available follow-up with a FT4 measurement, are presented in Table 1.

\section{Normalization of FT4 concentrations with respect to different laboratory reference ranges}

\section{Available laboratory reference ranges of the FT4 measurements and PNA dependent target reference ranges}

Each of the measured FT4 values in our dataset is accompanied by a corresponding laboratory reference range. These ranges are PNA dependent but also assay- and center-related. In total, 34 different FT4 laboratory reference ranges were identified for the 505 measurements. Observed laboratory reference ranges from all FT4 measurements over time are shown in Fig. 2. We observe that during the first 30 days, few upper limits of the reference ranges are unusually large.

To merge all FT4 measurements from the four clinical centers, a normalization method was constructed based on PNA dependent target reference ranges taken from Kapelari et al. [25], see Table 2 for the 2.5 and 97.5 percentiles and Fig. 2. After normalization, the FT4 values can be treated as if they were obtained from a single standard laboratory [26].

\section{Construction of a time-dependent normalization method during treatment}

A normalization method is based on statistical assumptions which have to be verified prior to application. We observed that the distribution of FT4 measurements changes during treatment in our $\mathrm{CH}$ population. At time of diagnosis, nonnormalized FT4 measurements follow a right-skewed distribution with several values close to zero, representing the disease severity in our cohort of severe, moderate and mild forms (Fig. 3a). However, during treatment, the distribution transforms towards normality, as shown in Fig. 3b-d for different time points and intervals. Since successfully treated patients have FT4 values in the healthy range, such a distribution is expected.

We denote with $x_{\text {meas }}$ the performed FT4 measurement and with $r_{l o w}$ and $r_{u p}$ the value of the lower and upper 

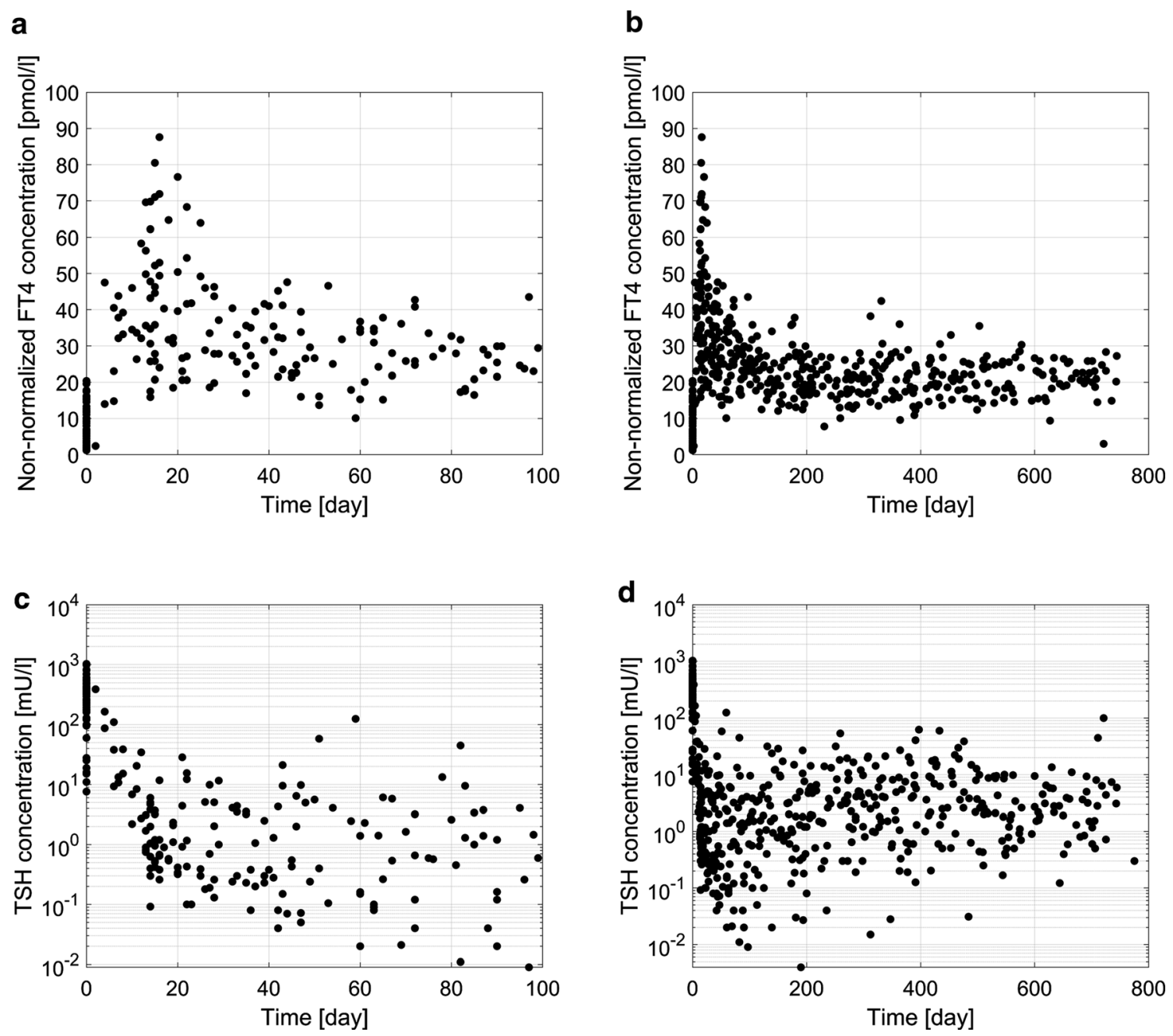

Fig. 1 Non-normalized FT4 measurements $(n=505)$ are shown in panel $\mathbf{a}$ and $\mathbf{b}$. TSH measurements $(\mathrm{n}=510)$ are shown in panel $\mathbf{c}$ and $\mathbf{d}$

limits of the laboratory reference range associated with this measurement. In addition, $r_{\text {low }}^{\text {Std }}$ and $r_{u p}^{\text {Std }}$ denote the lower (2.5 percentile) and upper (97.5 percentile) limits of the PNA dependent target reference range taken from Table 2.

Karvanen [26] proposed and derived for right-skewed distributions a scale normalization formula to compute the normalized FT4 value $x_{\text {norm }}$ :

$x_{\text {norm }}=x_{\text {meas }} \cdot \frac{r_{u p}^{S t d}}{r_{u p}}$

For normally distributed data, it was shown that the Chuang-Stein formula [27], also called location-scale normalization formula, $x_{\text {norm }}=\left(x_{\text {meas }}-r_{\text {low }}\right) \cdot \frac{r_{\text {up }}^{S t d}-r_{\text {low }}^{S t d}}{r_{\text {up }}-r_{\text {low }}}+r_{\text {low }}^{S t d}$

is valid. For more details and justification of Eqs. (1) and (2) see [26].

Since the FT4 distribution transforms from right-skewed to normal during treatment, we propose a combination of the scale and location-scale formula. More precisely, for small times $t$ the scale formula is dominant. For increasing times $t$ up to a certain time threshold $t_{s}$, the scale formula transforms into a mixture of scale and location-scale formula. After the threshold $t_{s}$, only the location-scale formula is applied: 
$x_{\text {norm }}=\left\{\begin{array}{l}\frac{t}{t_{s}} \cdot\left(\left(x_{\text {meas }}-r_{\text {low }}\right) \cdot \frac{r_{u p}^{S t d}-r_{\text {low }}^{S t d}}{r_{\text {up }}-r_{\text {low }}}+r_{\text {low }}^{S t d}\right)+\left(1-\frac{t}{t_{s}}\right) \cdot x_{\text {meas }} \cdot \frac{r_{\text {up }}^{S t d}}{r_{\text {up }}} \quad \text { for } t \leq t_{s} \\ \left(x_{\text {meas }}-r_{\text {low }}\right) \cdot \frac{r_{u p}^{S t d}-r_{\text {low }}^{S t d}}{r_{\text {up }}-r_{\text {low }}}+r_{\text {low }}^{\text {Std }} \quad \text { for } t>t_{s}\end{array}\right.$

Table 1 Demographic and laboratory patient characteristics at start of LT4 treatment and at last available follow-up

\begin{tabular}{|c|c|c|c|}
\hline & Unit & Median [IQR] & [Min, Max] \\
\hline \multicolumn{4}{|c|}{ Patient parameters at start of treatment } \\
\hline $\mathrm{GA}^{\mathrm{a}}$ & week & $40.5[38.0,41.3]$ & {$[28.3,42.9]$} \\
\hline PNA & day & $7[6,9]$ & {$[3,231]$} \\
\hline Weight $^{\mathrm{b}}$ & $\mathrm{kg}$ & $3.3[2.9,3.8]$ & {$[0.95,8.07]$} \\
\hline $\mathrm{FT}^{\mathrm{c}}$ & $\mathrm{pmol} / \mathrm{l}$ & $7.0[3.4,12.3]$ & {$[1.2,20.4]$} \\
\hline $\mathrm{TSH}^{\mathrm{d}}$ & $\mathrm{mU} / \mathrm{l}$ & $267[146,430]$ & {$[7.6,1026]$} \\
\hline LT4 dose total daily & mcg/day & $25[25,37.5]$ & {$[10,50]$} \\
\hline LT4 dose per kg body weight ${ }^{\mathrm{f}}$ & $\mathrm{mcg} / \mathrm{kg} / \mathrm{day}$ & $8.8[6.8,13.02]$ & {$[2.8,28.4]$} \\
\hline \multicolumn{4}{|c|}{ Patient parameters at last available follow-up with FT4 measurement } \\
\hline PNA & day & $602[362,708]$ & {$[98,769]$} \\
\hline Weight $^{\mathrm{e}}$ & $\mathrm{kg}$ & $11.3[9.4,12.7]$ & {$[4.3,15]$} \\
\hline FT4 & $\mathrm{pmol} / \mathrm{l}$ & $21.0[18.4,25.6]$ & {$[3,35.5]$} \\
\hline TSH & $\mathrm{mU} / 1$ & $2.2[0.90,4.7]$ & {$[0.005,100]$} \\
\hline LT4 dose total daily & $\mathrm{mcg} /$ day & $50[37.5,50]$ & {$[15,75]$} \\
\hline LT4 dose per kg body weight ${ }^{\mathrm{f}}$ & $\mathrm{mcg} / \mathrm{kg} / \mathrm{day}$ & $3.8[3.4,4.4]$ & {$[1.8,6.7]$} \\
\hline
\end{tabular}

${ }^{\mathrm{a}} 13$ values missing; ${ }^{\mathrm{b}} 8$ measurements missing; ${ }^{\mathrm{c}} 5$ measurements missing; ${ }^{\mathrm{d}} 6$ measurements missing; ${ }^{\mathrm{e}} 6$ measurements missing; ${ }^{\mathrm{f}}$ computed based on imputed values for missing weight measurements a

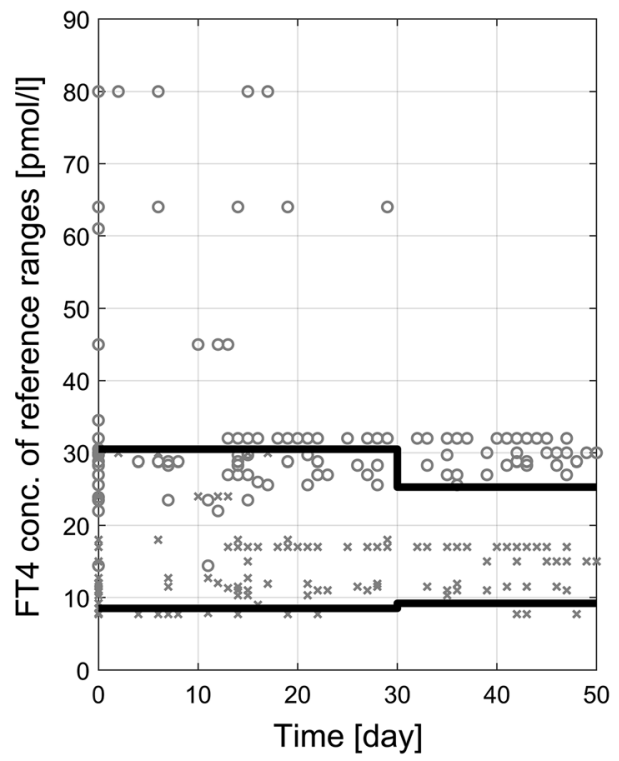

Fig. 2 Presentation of all available laboratory reference ranges of FT4 measurements over time. Grey circles denote the upper limit and grey crosses the lower limit of each range. Black lines show the upper and lower limits of the target reference range (compare Table 2), where

b

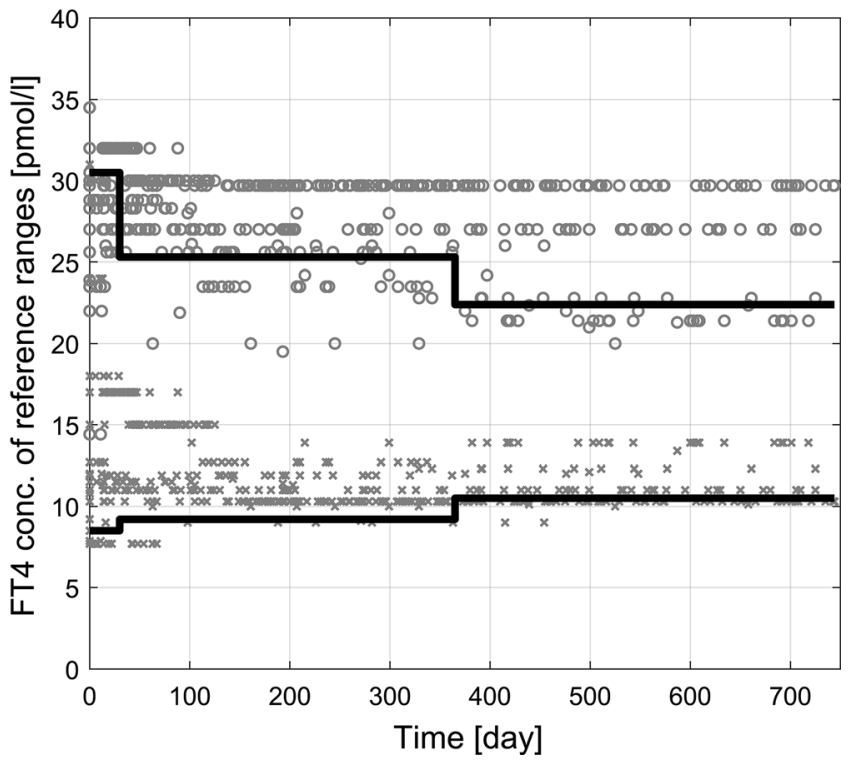

for simplicity in this Figure, individual PNA at start of treatment was neglected. The first 50 days are shown in panel a, and the total time interval is presented in panel $\mathbf{b}$ 
Table 2 Percentiles $(2.5,50$ and 97.5) of the target reference range for FT4 and TSH concentrations of neonates and infants based on postnatal age taken from [25]

\begin{tabular}{lcccccr}
\hline & FT4 $(\mathrm{pmol} / \mathrm{l})$ & \multicolumn{5}{c}{ TSH (mU/l) } \\
\hline $\begin{array}{l}\text { Percentile } \\
\text { Postnatal age }\end{array}$ & 2.5 & 50 & 97.5 & 2.5 & 50 & 97.5 \\
$0-1$ months & 8.50 & 20.10 & 30.50 & 0.70 & 3.50 & 18.10 \\
$1-12$ months & 9.17 & 15.50 & 25.28 & 1.12 & 2.85 & 8.21 \\
$1-5$ years & 10.45 & 15.70 & 22.35 & 0.80 & 2.70 & 6.26 \\
\hline
\end{tabular}

\section{PMX model to characterize FT4 based on remaining endogenous T4 production and exogenous LT4 treatment}

\section{Part I: structural PMX model for exogenous LT4 treatment}

Oral exogenous LT4 mcg/day administration is characterized with the input function $\operatorname{In}\left(t_{j}, \operatorname{dos}_{j}, F\right)$, where $t_{j}$ is the dosing time point, dose $_{j}$ the total daily LT4 dose mcg/day and $F$ the bioavailability. The dose was converted with factor 1.29 to $\mathrm{nmol} /$ day (molecular weight of thyroxine $776.9 \mathrm{~g} / \mathrm{mol}$ ). The absorption compartment $A_{B}$ nmol reads

$\frac{d}{d t} A_{B}=\operatorname{In}\left(t_{j}, \operatorname{dose}_{j}, F\right)-k_{a} \cdot A_{B}, \quad A_{B}(0)=0$

with absorption rate $k_{a}$.

\section{Part II: structural PMX model for endogenous T4 production}

We apply a typical one-compartment pharmacokinetic model with linear first-order elimination and further assume that a remaining endogenous $\mathrm{T} 4$ production is present, described with the zero-order production rate $k_{\text {endo }}$ $\mathrm{nmol} / \mathrm{day}$

$\frac{d}{d t} A_{C}=k_{a} \cdot A_{B}+k_{\text {endo }}-k_{\text {el }} \cdot A_{c}, \quad A_{C}(0)=\frac{k_{\text {endo }}}{k_{\text {el }}}$

where $A_{C}$ characterizes the central compartment with the amount of T4 nmol. Linear elimination is described with the first-order elimination rate $k_{e l} 1 /$ day and the initial
Fig. 3 Change of distribution of the non-normalized FT4 measurements from a rightskewed distribution towards a normal distribution for increasing time of treatment. Panel a shows the distribution at start of treatment $t=0$ of all available FT4 measurements, panel $\mathbf{b}$ and $\mathbf{c}$ show the distribution for later time intervals, whereas panel d shows the distribution based on the individual last measurement time point
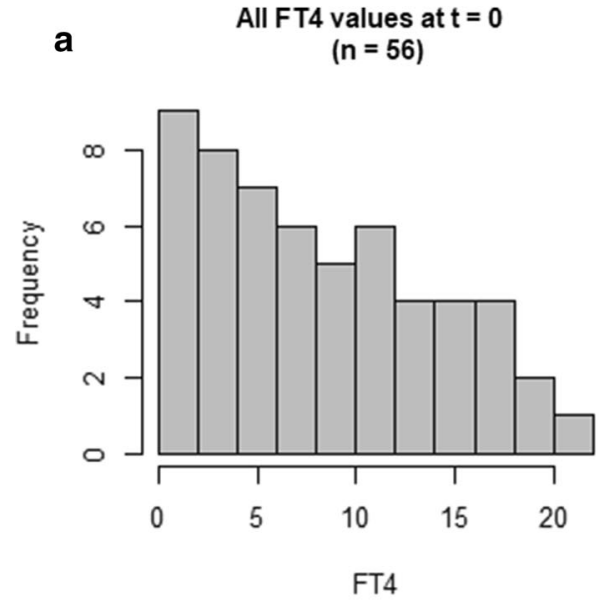

C

All FT4 values with $t>=100$ and $t<150$ ( $n=38$ )

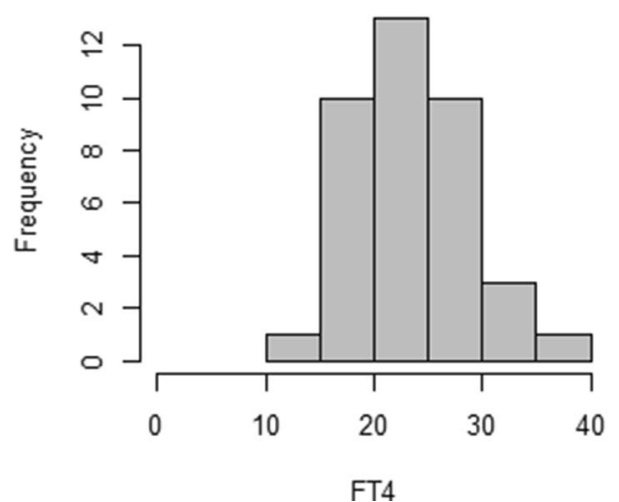

b

All FT4 values with $t>0$ and $t<50$ $(n=109)$

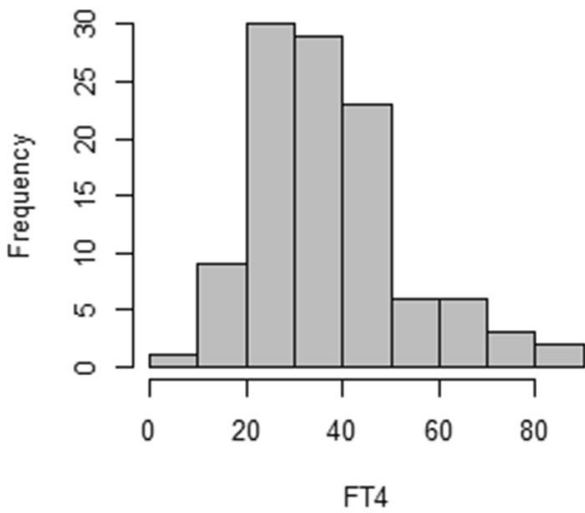

d All FT4 values at last time point ( $n=61$ )

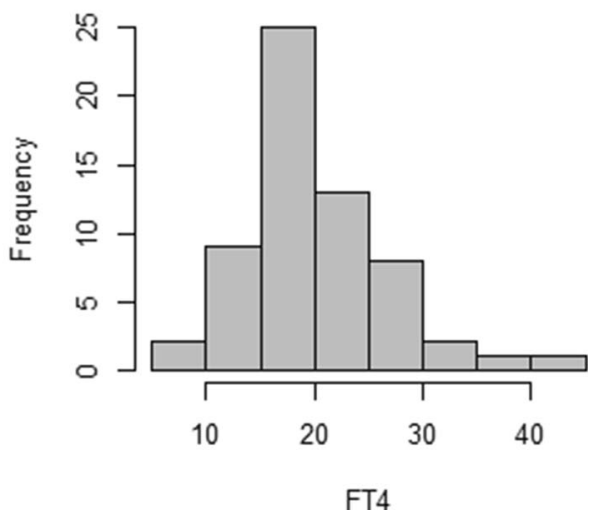


condition assumes that the endogenous T4 production is in an equilibrium prior to treatment. This assumption might not be fully correct at start of treatment since the neonate may still slightly benefit from transplacental passage of maternal hormones until birth. However, after stopping the treatment the amount of $\mathrm{T} 4$ will always return to the equilibrium $\frac{k_{e n d o}}{k_{e l}}$ given in Eq. (5) which is consistent with the assumption that the disease cannot be cured.

\section{Part III: structural PMX model with body weight development}

The FT4 concentration is derived by transforming the T4 unit nmol to pmol and assuming that the FT4 concentration corresponds to $0.03 \%$ of T4 [28]. Therefore, FT4 concentration $\mathrm{pmol} / \mathrm{l}$ reads

$C_{F T 4}=0.3 \cdot \frac{A_{C}}{V(W)}$

where $V 1$ is the volume of distribution depending on current body weight $\mathrm{kg} W(t)$ :

$V(W)=f_{V} \cdot\left(\frac{W(t)}{W_{\text {Ref }}}\right)^{\beta_{W}}$

where $f_{V} 1$ is a multiplicative factor relating body weight with volume of distribution, $W_{R e f}$ is the median of body weight of the underlying population, and $\beta_{W}$ the power exponent. Obviously, if the treatment stops, FT4 concentration will return to an equilibrium which depends on the current body weight.

\section{Interpolation of body weight and TSH over time}

In total, $\mathrm{n}=474$ measurements of body weight $W$ were available. Daily values for body weight and TSH were imputed by non-linear interpolation.

\section{Covariate modeling}

Categorical (not time-varying) covariates were implemented and tested with the default setting from The Monolix Suite 2020R1 (Lixoft, Orsay, France). Continuous covariates were tested at model parameters with the power model

$\theta_{i}=\theta_{\text {pop }} \cdot\left(\frac{\operatorname{Cov}_{i}}{\operatorname{Cov}_{R e f}}\right)^{\beta}$

where $\theta_{i}$ is the individual model parameter, $\theta_{\text {pop }}$ is the population model parameter (typical value), $\operatorname{Cov}_{i}$ is the individual (possibly time-varying) covariate, $\operatorname{Cov}_{R e f}$ is the reference covariate value and $\beta$ is the exponent describing the covariate effect.

\section{Covariate selection and testing}

Covariates to be tested were selected based on completeness, possible correlations among each other and clinical plausibility.

GA was not available for all patients, however strongly correlates with birth weight, and was therefore not tested. PNA at start of treatment (continuous) and sex (categorical) were included in covariate testing.

Body weight over time (time-varying) with the interpolated values for missing measurements was already incorporated in the model to compute the volume of distribution, compare Eqs. (6) and (7). Body weight at start of treatment (continuous) was not selected for testing because 8 measurements were missing and body weight over time was already included.

TSH effects over time on the endogenous production rate were tested with two different groupings. In the first group, TSH values were categorized as follows: category 1 with $\mathrm{TSH}<3$ $\mathrm{mU} / \mathrm{l}$ (milliunits per liter) and category 2 with TSH $\geq 3 \mathrm{mU} / \mathrm{l}$. The value $3 \mathrm{mU} / \mathrm{l}$ was selected since it corresponds to the average of the median target reference range in our study population, compare Table 2 . In the second group, TSH values were split into three categories: category $1 \mathrm{TSH}<1 \mathrm{mU} / \mathrm{l}$, category $2 \mathrm{TSH} \geq 1$ and $\mathrm{TSH}<10 \mathrm{mU} / \mathrm{l}$, and category 3 $\mathrm{TSH} \geq 10 \mathrm{mU} / \mathrm{l}$. Chosen values roughly correspond to the average of the lower and upper limits of the target reference range, compare Table 2. Although it is obvious that a relationship between TSH at start of treatment (continuous) and FT4 at start of treatment exists, TSH at start of treatment was not selected because 6 measurements were missing.

\section{Statistical data presentation and applied software}

All laboratory and demographic values are reported as median together with the interquartile range (IQR) $[25 \%$, $75 \%$ ]. Descriptive statistical analysis was performed in $\mathrm{R}$ 3.6.0 ( $\mathrm{R}$ core team, Vienna, Austria) and hypothesis testing was executed with the Student's t-test for normally distributed values and with the Wilcox rank test for non-normally distributed values. Non-linear mixed-effects modeling was performed in The Monolix Suite 2020R1 (Lixoft, Orsay, France). A-posteriori data visualization was implemented in R or Matlab 2020a (MathWorks, Natick, MA, USA).

\section{Results}

The Results section consists of five paragraphs. First, the non-normalized and normalized measurements are compared. Second, model parameter estimates are presented. 
Table 3 Comparison of non-normalized and normalized FT4 values for different time points and time intervals

\begin{tabular}{lccc}
\hline Time points/intervals (day) & Non-normalized FT4 (pmol/l) Median $[\mathrm{IQR}]$ & Normalized FT4 (pmol/l) Median [IQR] & Significance \\
\hline Start of treatment $\left(t_{0}=0\right)$ & $7.0[3.4,12.3]$ & $5.2[2.4,10.4]$ & N.s. $^{\mathrm{a}}$ \\
$0<t<50$ & $33.6[26.3,45.2]$ & $31.7[22.6,43.2]$ & N.s. $^{\mathrm{a}}$ \\
$100 \leq t<150$ & $23.4[19.5,26.3]$ & $20.6[18.0,24.1]$ & N.s. $^{a}$ \\
Last available time point & $21.0[18.4,25.6]$ & $18.0[16.0,22.7]$ & N.s. $^{a}$ \\
\hline
\end{tabular}

${ }^{\mathrm{a}}$ Not significant

Third, a TSH feedback effect on FT4 is tested. Fourth, the final PMX model for $\mathrm{CH}$ is presented. Fifth, additional tests are performed.

\section{Comparison of non-normalized and normalized measurements}

Transition from a right-skewed towards a normal distribution was expected at time threshold $t_{s}=150$ day, compare Fig. 3. After application of the proposed scale/ location-scale formula Eq. (3), we observe that normalized FT4 values are slightly but not significantly lower than non-normalized measurements. Comparison for start of treatment $\left(t_{0}=0\right)$, additional time intervals $0<t<50$, $100 \leq t<150$, and at $t=$ last available time point are presented in Table 3.

We emphasize that the application of the Chuang-Stein (location-scale) formula alone resulted in 29 negative values for FT4 at start of treatment. This was expected since the normal assumption was violated (compare Fig. 3a) and laboratory reference ranges were quite large compared to the actual FT4 value.

TSH over time is a highly variable and sensitive marker with absolute values ranging from the limit of quantification up to $1026 \mathrm{mU} / \mathrm{l}$ in our study population, compare Fig. 1b. An investigation of the distributions of TSH at specific time points showed mostly right-skewed behavior with no clear tendency towards normal (data not shown). In total, 43 TSH measurements were not accompanied by laboratory reference ranges (incomplete or missing) and therefore, TSH was not normalized.

\section{PMX model parameter estimation based on normalized FT4 values}

LT4 is variably absorbed and bioavailability is reported between 40 to $80 \%$ (https://go.drugbank.com/drugs/ DB00451). We fixed the bioavailability to $F=0.6$ in Eq. (4). The model Eqs. (4)-(7) consists of the structural model parameters $k_{a}, k_{\text {endo }}, f_{V}$ and $k_{e l}$. The absorption rate $k_{a}$ was fixed to $201 /$ day, realizing a maximal FT4 peak at $2 \mathrm{~h}$ [29], and had no inter-individual variability (IIV). The endogenous production rate $k_{\text {endo }}$, the factor $f_{V}$ relating body weight with volume of distribution, as well as the power exponent $\beta_{W}$ were estimated with a log-normal distribution. Based on available data, the elimination rate $k_{e l}$ is difficult to estimate. Since T4 half-life in plasma is on average 7 days [29], $k_{e l}$ was fixed to 0.1 1/day. Allowing IIV for $k_{e l}$ resulted in a high shrinkage based on the individual conditional mode estimations and a small standard deviation of the random effects. Hence, IIV on this parameter was omitted. We remark that a formulation of the model in terms of clearance with a weight-based allometric scaling approach was omitted because of (i) insufficient FT4 data to reasonably apply such an approach, (ii) the lack of information regarding maturation effect on the half-life in literature, and (iii) the rather small weight range. Data fitting was performed with a proportional residual error model.

PNA at start of treatment showed a significant but weak effect on the endogenous production rate $k_{\text {endo }}$. However, the PNA effect was exclusively driven by 4 patients with PNAs larger than 50 days. Without these 4 patients, no significant effect was available. Therefore, the PNA effect was not included in the final model. Sex had no effect on any model parameter.

\section{Test for TSH feedback effects on FT4}

Low FT4 (or T4) values cause increased TSH levels which in turn will try to stimulate the FT4 (or T4) production. Therefore, we tested a TSH feedback on the endogenous T4 production rate $k_{\text {endo }}$. More precisely, $k_{\text {endo }}$ is modeled as a function of TSH and changes over time, denoted by $k_{\text {endo,Cov }}$. Hence, $k_{\text {endo }}$ is now substituted by $k_{\text {endo,Cov }}$ in Eq. (5). First, the previously defined two time-varying categorical TSH groups were tested. For the first grouping we applied

$k_{\text {endo }, \text { Cov }}= \begin{cases}k_{\text {endo }} & \text { for category } 1 \\ k_{\text {endo }}+\beta_{1} & \text { for category } 2\end{cases}$

and for the second grouping: 
$k_{\text {endo }, \text { Cov }}=\left\{\begin{array}{lr}k_{\text {endo }} & \text { for category } 1 \\ k_{\text {endo }}+\beta_{1} \text { for category } 2 \\ k_{\text {endo }}+\beta_{2} \text { for category } 3\end{array}\right.$

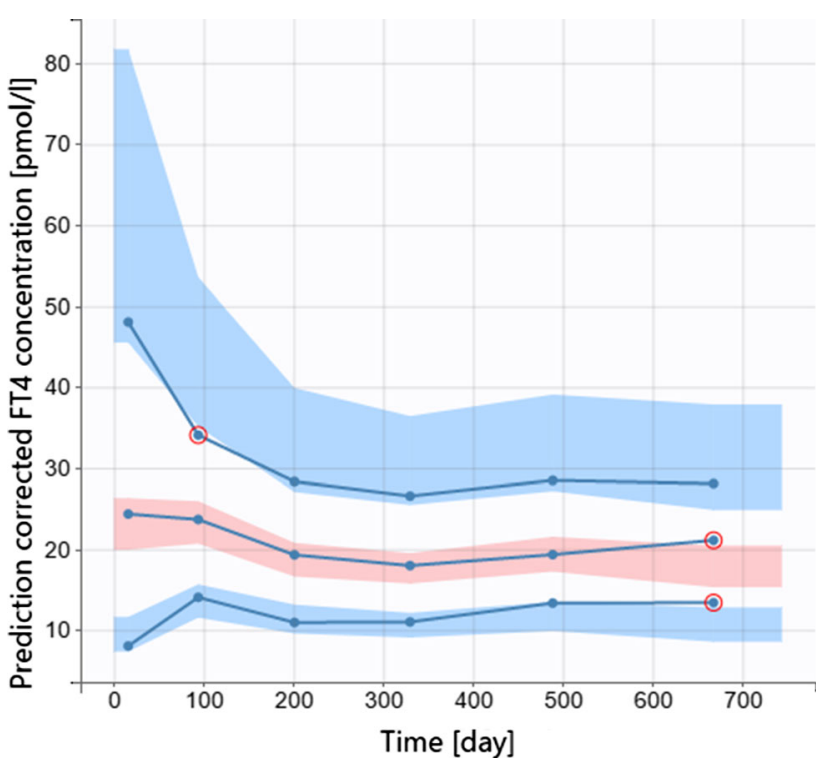

Second, the effect of actual TSH measurements on $k_{\text {endo }}$ were tested with

$k_{\text {endo }, \text { Cov }}=k_{\text {endo }} \cdot f(T S H)$

and

$k_{\text {endo }, \text { Cov }}=k_{\text {endo }}+\beta_{3} \cdot f(T S H)$

The function $f(T S H)$ was either (i) $f(T S H)=T S H$, (ii)

$f(T S H)=\left(\frac{T S H}{T S H_{R e f}}\right)^{\beta}$, (iii) $f(T S H)=\log (T S H)$, or

$f(T S H)=\left(\frac{\log (T S H)}{\log \left(T S H_{\text {Ref }}\right)}\right)^{\beta}$.

Based on the available data, it was difficult to observe an improvement in data fitting by including a TSH feedback on the endogenous production rate. With a combined assessment of typical data fitting criteria such as goodnessof-fit plots, reduction of the objective function, reduction of variability in random effects, and changes in standard error, no overall improvement could be concluded. Therefore, TSH feedback effect was not included in the model.

\section{Final PMX model for CH based on normalized FT4 values}

The final model consists of Eqs. (4)-(7) without any additional covariate effects. Final model parameter estimates are shown in Table 4. Visual predictive check is presented in Fig. 4. Other goodness-of-fit plots and individual profiles are available in the supplemental material.

Fig. 4 Visual predictive check based on normalized FT4 measurements modeled with Eqs. (4)-(7)

\section{Additional tests}

In the following, an additional test was performed to further verify the final PMX model Eqs. (4)-(7).

\section{Comparison of results with parameter estimates from non- normalized data}

With the final model Eqs. (4)-(7), the non-normalized FT4 data were fitted and individual parameter values were compared. The estimated individual parameter values for the endogenous production rate $k_{\text {endo }}$ for non-normalized FT4 values were significantly higher $(\mathrm{p}<0.005)$ than for normalized values. Comparison of estimated individual

Table 4 Population estimates (typical values), standard deviation of the random effects and additional parameters obtained from fitting normalized FT4 data with the final PMX model Eqs. (4)-(7)

\begin{tabular}{llll}
\hline Parameter name & Description & Unit & Estimate (r.s.e. ${ }^{\mathrm{a}}$ ) \\
\hline $\begin{array}{l}\text { Population estimates (fixed effects) } \\
k_{a}\end{array}$ & Absorption rate & & \\
$k_{e l}$ & Elimination rate & $1 /$ day & $20 \mathrm{fix}$ \\
$f_{V}$ & Multiplicative factor & $1 /$ day & $0.1 \mathrm{fix}$ \\
$\beta_{W}$ & Power exponent & 1 & $4.96(3.7)$ \\
$k_{\text {endo }}$ & Endogenous production rate & - & $0.753(6.6)$ \\
Standard deviation of the random effects & nmol/day & $3.66(15.7)$ \\
$\omega_{f_{V}}$ & & \\
$\omega_{\beta_{W}}$ & & $0.249(11.9)$ \\
$\omega_{k_{\text {endo }}}$ & & $0.404(15.8)$ \\
Additional parameters and values & & $1.12(11.1)$ \\
Prop. residual error & & \\
$-2 \mathrm{LL}$ value & & 3268 \\
\hline
\end{tabular}

${ }^{\mathrm{a}}$ Relative standard error 
parameter values for $f_{V}$ and $\beta_{W}$ is more complicated since the volume of distribution depends on both parameters as given in Eq. (7). Hence, for any individual we have to consider the pair $\left(f_{V}, \beta_{W}\right)$ and compare the resulting volume of distribution obtained from non-normalized FT4 values to the resulting volume of distribution obtained from normalized FT4 values. We restrict the comparison to the volume of distribution at baseline and the volume of distribution for median body weight. In both situations, we observe that the volume of distribution for non-normalized FT4 values is significantly lower $(p<0.005)$ than for normalized data. Hence, these comparisons coincide with the fact that normalized FT4 data were on average lower than non-normalized FT4 data, compare Table 3.

\section{Discussion}

In this section we discuss the key findings in the context of the three main goals of this research project: (i) Presentation of challenges associated with a retrospectively collected dataset including long-term follow-up of 61 newborns and infants with a rare disease $(\mathrm{CH})$. (ii) Derivation of a time-dependent scale / location-scale normalization method to account for the multi-center/-assay nature of the data. (iii) Development of a practical PMX model describing FT4 concentration and LT4 treatment.

The first goal of this article was to describe retrospectively collected multi-center data from newborns and infants with $\mathrm{CH}$ up to 2 years. Clinical and laboratory records from children with a rare disease are stored for approximately 20 years as such patients were followed from birth until transition to an adult endocrinologist and the retention requirement is 10 years in Switzerland. This allowed for collecting data from patients ranging back to 1995 resulting in $\mathrm{n}=61$ newborns with $\mathrm{CH}$ after application of inclusion and exclusion criteria. Assuming approximately 80,000 live births per year in Switzerland in the last decades (https://www.bfs.admin.ch) leads to the total number of 1.8 million live births from 1995 to 2018 . The incident rate of $\mathrm{CH}$ in Switzerland is 1:3500 (https:// www.neoscreening.ch) resulting in approximately 515 newborns with $\mathrm{CH}$ between 1995 and 2018. Hence, our dataset roughly consists of $12 \%$ of all newborns with $\mathrm{CH}$ in Switzerland from 1995 to 2018. The dataset revealed the major problem of strongly variable FT4 laboratory reference ranges especially in the first weeks of life. There are several reasons for this. First, some reference ranges were simply adult references not taking into account the higher normative values in the neonates and young infants, while other reference ranges were adapted to the neonatal and infant age group. Second, during long-term observation, laboratory assays and consequently reference ranges changed for the same parameter within a center. Third, different laboratory methods were used at different centers and changed at different time points. Harmonization and normalization of the clinical dataset was an important part of the work before any modeling of the disease and the therapy could have been done. While prospectively planned studies allow avoiding such technical hurdles, in rare diseases with a limited number of patients at a single center and even in a region or country, the retrospective approach has the advantage to establish datasets for generating hypotheses and modeling in parallel to the realization of a prospective study. In summary, retrospective data analyses are feasible even in the context of, as in our case, 34 different laboratory reference ranges, if normative data standardization is implemented.

The second goal was to develop a normalization method. However, before developing such a method, we have to discuss the well-known question of whether normalization is indeed necessary. The answer depends on the particular situation. On one hand, in drug development, different reference ranges can usually be avoided by applying standardized assays in the same laboratories right from the beginning of the study. If this standardization is not possible, it is suggested to ignore laboratory differences and "the analyst must accept the data as is" [30] or consider the application of normalization methods "as the last resort" [31]. On the other hand, our multi-assay/-center dependent nature of the measurements, as previously explained, calls for a normalization method to reasonably merge available data. The Chuang-Stein formula is a linear approach to normalize data with different reference ranges. Hence, normally distributed data will remain normally distributed after application of the Chuang-Stein formula. However, the Chuang-Stein formula can produce negative normalized values in specific situations. This issue was reported by Chuang-Stein [31] and was also observed when applying the Chuang-Stein formula to our FT4 measurements. Another issue is the assumption of normality itself which is not fulfilled at start of treatment as shown for our FT4 measurements. For such right-skewed distributed data, Karvanen [26] derived a scale formula for normalization. Therefore, we developed a time-dependent normalization method that is based on the scale formula in the beginning of treatment and transitions to the location-scale formula for normally distributed data when the FT4 values are in the healthy range.

The third goal was to develop a practical PMX model for LT4 treatment to characterize FT4 measurements. The major aim was that the PMX model can be applied in a clinical setting. Therefore, the PMX model has to be in a fair balance between the physiological mechanism and the capability to be applied to routinely collected clinical data. Already in the 1950s, Danziger et al. [18] published two 
systems of non-linear differential equations that describe the thyroid-pituitary homeostatic mechanism and performed a mathematical analysis of the model structures. Notable from our perspective is the work by Mak et al. [19] who developed a model for hypothyroidism consisting of 6 compartments describing $\mathrm{T} 3$ and $\mathrm{T} 4$ concentration in plasma, extravascular tissue and general tissue, and 17 model parameters. Other models taking complex details into account to characterize interactions in the HPT axis were developed by Leow [32], Degon et al. [33], or Eisenberg et al. [21]. Mukhopadhya et al. [34] and Berberich et al. [35] even applied delay differential equations [36] to capture existing time delays in the interactions. Remarkable is the systems pharmacology model by Ekerot et al. [22] based on pharmacokinetic/pharmacodynamic concepts to describe the impact of thyroperoxidase inhibition in dogs where model parameters are estimated with non-linear mixed-effects modeling. On one hand, all of these models offer great insights into the detailed mechanism of the HPT axis but they are on the other hand difficult to apply to clinical data due to their complexity. Development of an applicable and predictive mathematical model for clinical practice is a tradeoff between available data, prior knowledge from literature, complexity of the underlying physiological and pathophysiological mechanisms, and, most importantly, the capability to address clinically relevant research questions. The final structure of our developed PMX model for FT4 measurements and LT4 treatment was a one-compartment model with absorption and an additional zero-order endogenous production rate. The endogenous production rate of $\mathrm{T} 4$ allows testing for TSH feedback effects. More precisely, low FT4 levels cause increased TSH levels which in turn should stimulate FT4 production. TSH is a highly variable biomarker for thyroid functionality. However, it is unclear how strong the TSH feedback effect is on the FT4 production in $\mathrm{CH}$ patients. We tested different mathematical terms to estimate the effect of TSH concentration on the endogenous production rate of $\mathrm{T} 4$ but could not identify a significant effect based on (i) estimated model parameters, (ii) reduction of objective function value, and (iii) diagnostic plots. The following three reasons may explain these unexpected results. First, the cause of the disease is unresponsiveness or reduced responsiveness of the thyroid gland to produce FT4 to even high levels of TSH as seen at the FT4 and TSH values at diagnosis. Thus, the expected stimulatory effect of TSH is not or clearly less efficient in patients with $\mathrm{CH}$ than in healthy individuals. Second, only 52 of 505 measurements represent pairs of TSH and FT4 before start of LT4 treatment and an effect would only be found gradually in the moderate to mild forms further reducing the numbers. Third, after initiation of substitutive treatment with LT4, TSH is falling back into the target reference range as the result of a normally functioning negative feedback loop to exogenously administered LT4 on TSH synthesis and secretion. Further, TSH remains in the target reference range during long-term treatment, if the dose is ideally adapted to the needs of the growing child. Lack of modeling a feedback from TSH on FT4 should not be overestimated since it makes the developed PMX model even more applicable to the clinical setting, where TSH measurements might be missing.

In summary, this research article discussed challenges in analyzing retrospective data from pediatric patients with a rare disease and presented a practical and clinically useful PMX model that characterizes FT4 concentration under LT4 treatment in newborns and infants with $\mathrm{CH}$.

Supplementary Information The online version contains supplementary material available at https://doi.org/10.1007/s10928021-09765-w.

Open Access This article is licensed under a Creative Commons Attribution 4.0 International License, which permits use, sharing, adaptation, distribution and reproduction in any medium or format, as long as you give appropriate credit to the original author(s) and the source, provide a link to the Creative Commons licence, and indicate if changes were made. The images or other third party material in this article are included in the article's Creative Commons licence, unless indicated otherwise in a credit line to the material. If material is not included in the article's Creative Commons licence and your intended use is not permitted by statutory regulation or exceeds the permitted use, you will need to obtain permission directly from the copyright holder. To view a copy of this licence, visit http://creativecommons. org/licenses/by/4.0/.

Funding Open Access funding provided by Universität Basel (Universitätsbibliothek Basel). This study was supported by a Grant awarded to GK from the Swiss National Science Foundation; Grant No. 179510. This work has been supported by the DFG, Project No. SCHR 692/3-1 (Germany).

\section{References}

1. Food and Drug Administration. https://rarediseases.info.nih.gov/ diseases/pages/31/faqs-about-rare-diseases

2. Richter T, Nestler-Parr S, Babela R et al (2015) Rare disease terminology and definitions-a systematic global review: report of the ISPOR rare disease special interest group. Value Health 18:906-914

3. Koch G, Schropp J (2014) Mathematical concepts in pharmacokinetics and pharmacodynamics with application to tumor growth. In: Kloeden P, Poetzsche C (eds) Nonautonomous dynamical systems in the life sciences lecture notes in mathematics. Springer, Cham

4. Pfister M, D'Argenio DZ (2010) The emerging scientific discipline of pharmacometrics. J Clin Pharmacol 50:6S

5. van Trotsenburg P, Stoupa A, Léger J et al (2021) Congenital Hypothyroidism: A 2020-2021 Consensus Guidelines Update-An ENDO-European Reference Network Initiative Endorsed by the European Society for Pediatric Endocrinology and the European Society for Endocrinology. Thyroid. 31(3):387-419. https://doi. org/10.1089/thy.2020.0333 
6. Szinnai G (2014) Clinical genetics of congenital hypothyroidism. Endocr Dev 26:60-78

7. Illig R, Largo RH, Qin Q, Torresani T, Rochiccioli P, Larsson A (1987) Mental development in congenital hypothyroidism after neonatal screening. Arch Dis Child 62:1050-1055

8. Glorieux J, Dussault J, Van Vliet G (1992) Intellectual development at age 12 years of children with congenital hypothyroidism diagnosed by neonatal screening. J Pediatr 121:581-584

9. Dubuis JM, Glorieux J, Richer F, Deal CL, Dussault JH, Van Vliet G (1996) Outcome of severe congenital hypothyroidism: closing the developmental gap with early high dose levothyroxine treatment. J Clin Endocrinol Metab 81:222-227

10. Bongers-Schokking JJ, de Muinck Keizer-Schrama SM (2005) Influence of timing and dose of thyroid hormone replacement on mental, psychomotor, and behavioral development in children with congenital hypothyroidism. J Pediatr 147:768-774

11. Szinnai G, Polak M (2021) The maturation of thyroid function in the fetus, in the perinatal period and during childhood. In: Braverman E, Cooper D, Kopp P (eds) Werner \& Ingbar's the thyroid: a fundamental and clinical text. Wolters Kluwer, Philadelphia, pp 839-854

12. Bakker B, Kempers MJ, De Vijlder JJ et al (2002) Dynamics of the plasma concentrations of TSH, FT4 and T3 following thyroxine supplementation in congenital hypothyroidism. Clin Endocrinol (Oxf) 57:529-537

13. Aleksander PE, Bruckner-Spieler M, Stoehr AM et al (2018) Mean high-Dose 1-thyroxine treatment is efficient and safe to achieve a normal IQ in young adult patients with congenital hypothyroidism. J Clin Endocrinol Metab 103:1459-1469

14. Dimitropoulos A, Molinari L, Etter K et al (2009) Children with congenital hypothyroidism: long-term intellectual outcome after early high-dose treatment. Pediatr Res 65:242-248

15. Alvarez M, Iglesias Fernandez C, Rodriguez Sanchez A, Dulin Lniguez E, Rodriguez Arnao MD (2010) Episodes of overtreatment during the first six months in children with congenital hypothyroidism and their relationships with sustained attention and inhibitory control at school age. Horm Res Paediatr 74:114-120

16. Bongers-Schokking JJ, Resing WC, de Rijke YB, de Ridder MA, de Muinck Keizer-Schrama SM (2013) Cognitive development in congenital hypothyroidism: is overtreatment a greater threat than undertreatment? J Clin Endocrinol Metab 98:4499-4506

17. Riggs DS (1952) Quantitative aspects of iodine metabolism in man. Pharmacol Rev 4:284-370

18. Danziger L, Elmergreen G (1956) The thyroid-pituitary homeostatic mechanism. Bull Math Biophys 18:1-13

19. Mak P, DiStefano J III (1978) Optimal control policies for the prescription of thyroid hormones. Math Biosci 42:159-186

20. Pandiyan B, Merrill SJ, Di Bari F, Antonelli A, Benvenga S (2018) A patient-specific treatment model for Graves' hyperthyroidism. Theor Biol Med Model 15:1

21. Eisenberg M, Samuels M, DiStefano JJ 3rd (2008) Extensions, validation, and clinical applications of a feedback control system simulator of the hypothalamo-pituitary-thyroid axis. Thyroid 18:1071-1085

22. Ekerot P, Ferguson D, Glamsta EL et al (2013) Systems pharmacology modeling of drug-induced modulation of thyroid hormones in dogs and translation to human. Pharm Res 30:1513-1524

23. Hoermann R, Eckl W, Hoermann C, Larisch R (2010) Complex relationship between free thyroxine and TSH in the regulation of thyroid function. Eur J Endocrinol 162:1123-1129

24. Hadlow NC, Rothacker KM, Wardrop R, Brown SJ, Lim EM, Walsh JP (2013) The relationship between TSH and free T(4) in a large population is complex and nonlinear and differs by age and sex. J Clin Endocrinol Metab 98:2936-2943

25. Kapelari K, Kirchlechner C, Hogler W, Schweitzer K, Virgolini I, Moncayo R (2008) Pediatric reference intervals for thyroid hormone levels from birth to adulthood: a retrospective study. BMC Endocr Disord 8:15

26. Karvanen J (2003) The statistical basis of laboratory data normalization. Drug Inf J 37:101-107

27. Chuang-Stein C (1992) Summarizing laboratory data with different reference ranges in multi-center clinical trials. Drug Inf J 26:77-84

28. Inada M, Kasagi K, Kurata S et al (1975) Estimation of thyroxine and triiodothyronine distribution and of the conversion rate of thyroxine to triiodothyronine in man. J Clin Invest 55:1337-1348

29. Colucci P, Yue CS, Ducharme M, Benvenga S (2013) A review of the pharmacokinetics of levothyroxine for the treatment of hypothyroidism. Eur Endocrinol 9:40-47

30. Bonate P (2006) Pharmacokinetic-pharmacodynamic modeling and simulation. Springer, New York

31. Chuang-Stein C (2001) Some issues concerning the normalization of laboratory data based on reference ranges. Drug Inf J 35:153-156

32. Leow M (2007) A mathematical model of pituitary-thyroid interaction to provide an insight into the nature of the thyrotropin-thyroid hormone relationship. J Theor Biol 248:275-287

33. Degon M, Chipkin SR, Hollot CV, Zoeller RT, Chait Y (2008) A computational model of the human thyroid. Math Biosci 212:22-53

34. Mukhopadhyay B, Battacharyya R (2006) A mathematical model describing the thyroid-pituitary axis with time delays in hormone transportation. Appl Math 51:549-564

35. Berberich J, Dietrich JW, Hoermann R, Muller MA (2018) Mathematical modeling of the pituitary-thyroid feedback loop: role of a TSH-T3-shunt and sensitivity analysis. Front Endocrinol (Lausanne) 9:91

36. Koch G, Krzyzanski W, Perez-Ruixo JJ, Schropp J (2014) Modeling of delays in PKPD: classical approaches and a tutorial for delay differential equations. J Pharmacokinet Pharmacodyn 41:291-318

Publisher's Note Springer Nature remains neutral with regard to jurisdictional claims in published maps and institutional affiliations.

\title{
Authors and Affiliations
}

\author{
Gilbert Koch $^{1}$ (D) Britta Steffens ${ }^{1} \cdot$ Stephanie Leroux ${ }^{1} \cdot$ Verena Gotta $^{1} \cdot$ Johannes Schropp ${ }^{2}$. \\ Pascal Gächter ${ }^{1,6} \cdot$ Freya Bachmann $^{2} \cdot$ Tatjana Welzel $^{1} \cdot$ Marco Janner $^{3} \cdot$ Dagmar L'Allemand $^{4}$. \\ Daniel Konrad $^{5}$ (D) Gabor Szinnai $^{6,7}$ (D) Marc Pfister ${ }^{1,7}$
}


$\triangle$ Gilbert Koch

Gilbert.Koch@ukbb.ch

1 Pediatric Pharmacology and Pharmacometrics, University Children's Hospital Basel UKBB, University of Basel, Basel, Switzerland

2 Department of Mathematics and Statistics, University of Konstanz, Konstanz, Germany

3 Pediatric Endocrinology, Diabetology and Metabolism, Department of Pediatrics, Bern University Hospital, University of Bern, Bern, Switzerland
Department of Pediatric Endocrinology and Diabetology, Children's Hospital of Eastern Switzerland, St. Gallen, Switzerland

5 Division of Pediatric Endocrinology and Diabetology and Children's Research Centre, University Children's Hospital, Zurich, Switzerland

6 Pediatric Endocrinology and Diabetology, University Children's Hospital Basel UKBB, University of Basel, Basel, Switzerland

7 Department of Clinical Research, University of Basel and University Hospital Basel, Basel, Switzerland 